Cochrane Database of Systematic Reviews

\title{
Art therapy for people with dementia (Review)
}

Deshmukh SR, Holmes J, Cardno A

Deshmukh SR, Holmes J, Cardno A.

Art therapy for people with dementia.

Cochrane Database of Systematic Reviews 2018, Issue 9. Art. No.: CD011073.

DOI: 10.1002/14651858.CD011073.pub2.

www.cochranelibrary.com 
TABLE OF CONTENTS

ABSTRACT 1

PLAIN LANGUAGE SUMMARY

SUMMARY OF FINDINGS

BACKGROUND

OBJECTIVES

METHODS

RESULTS

DISCUSSION

AUTHORS' CONCLUSIONS

ACKNOWLEDGEMENTS

REFERENCES

CHARACTERISTICS OF STUDIES

ADDITIONAL TABLES

APPENDICES

CONTRIBUTIONS OF AUTHORS

DECLARATIONS OF INTEREST

SOURCES OF SUPPORT

DIFFERENCES BETWEEN PROTOCOL AND REVIEW

INDEX TERMS 
[Intervention Review]

\section{Art therapy for people with dementia}

Sunita R Deshmukh¹, John Holmes², Alastair Cardno ${ }^{3}$

1London, UK. ${ }^{2}$ Leeds, UK. ${ }^{3}$ Academic Unit of Psychiatry and Behavioural Sciences, Leeds Institute of Health Sciences, University of Leeds, Leeds, UK

Contact: Sunita R Deshmukh, London, UK. sunitadeshmukh86@gmail.com.

Editorial group: Cochrane Dementia and Cognitive Improvement Group.

Publication status and date: New, published in Issue 9, 2018.

Citation: Deshmukh SR, Holmes J, Cardno A. Art therapy for people with dementia. Cochrane Database of Systematic Reviews 2018, Issue 9. Art. No.: CD011073. DOI: 10.1002/14651858.CD011073.pub2.

Copyright @ 2018 The Cochrane Collaboration. Published by John Wiley \& Sons, Ltd.

\section{A B S T R A C T}

\section{Background}

Art therapy is defined by the British Association of Art Therapists as: "a form of psychotherapy that uses art media as its primary mode of communication. Clients who are referred to an art therapist need not have experience or skill in art. The art therapist is not primarily concerned with making an aesthetic or diagnostic assessment of the client's image. The overall aim of its practitioners is to enable a client to change and grow on a personal level through the use of art materials in a safe and facilitating environment". Historically, drawings and paintings have been recognised as a useful part of therapeutic processes within psychiatric and psychological specialties, and this has been acknowledged within medical and neurology-based disciplines.

Arts-based therapies are generally considered as interventions managing manifestations of dementia, as they may help to slow cognitive deterioration, address symptoms related to psychosocially challenging behaviours and improve quality of life.

\section{Objectives}

To review the effects of art therapy as an adjunctive treatment for dementia compared with standard care and other non-pharmacological interventions.

\section{Search methods}

We identified trials from ALOIS - the Cochrane Dementia and Cognitive Improvement Group's Specialised Register - on 12 May 2014,20 March 2015, 15 January 2016, 4 November 2016, and 4 October 2017. We also handsearched the grey literature and contacted specialists in the field and authors of relevant reviews or studies to enquire about other sources of relevant information.

\section{Selection criteria}

All randomised controlled trials examining art therapy as an intervention for dementia.

\section{Data collection and analysis}

Two review authors independently extracted data. We examined scales measuring cognition, affect and emotional well-being, social functioning, behaviour and quality of life.

\section{Main results}

We found two studies that met the inclusion criteria, incorporating data on a total of 60 participants (from 88 randomised), in experimental groups $(n=29)$ and active control groups $(n=31)$. One study compared group art therapy with simple calculation activities over 12 weeks. The other study compared group art therapy with recreational activities over 40 weeks. It was not possible to pool the data for analysis from the included studies, due to heterogeneity in terms of differences in the interventions, control treatments and choice of outcome measures. 
In both studies there were no clear changes reported between the intervention group and the control group in the important outcome measures. According to GRADE ratings, we judged the quality of evidence for these outcome measures to be 'very low'.

\section{Authors' conclusions}

There is insufficient evidence about the efficacy of art therapy for people with dementia. More adequately-powered and high-quality studies using relevant outcome measures are needed.

\section{PLAIN LANGUAGE SUMMARY}

\section{Inconclusive findings on the effects of art therapy for people with dementia}

\section{Title}

Art therapy for people with dementia

\section{Background}

Dementia is a common condition that affects people's memory. It can also affect people's thinking, emotions and behaviour. Dementia has a major impact on health and society across the world. Some types of treatment other than medication may help people with dementia.

Art therapy is a type of psychological therapy, which means a treatment for problems of the mind and behaviour. Art can be used as a way to express and communicate thoughts and feelings. The aim of art therapists is to work with patients in ways that help them change and 'grow' on a personal level. This is done by using art materials in a safe environment that allows this process.

\section{Study Characteristics}

We looked at research trials of people with dementia doing art therapy, compared with usual care and other activities. We looked at effects of art therapy on memory and thinking, emotions, well-being, social behaviour and quality of life. We also looked at negative effects and costs of art therapy. We found two research studies with a total of 88 older people taking part. There were results for 60 people. One study compared groups doing art therapy or simple calculation activities over 12 weeks. The other study compared groups doing art therapy or recreational activities over 40 weeks. The evidence is current to October 2017.

\section{Key Results}

These two studies found no clear changes in memory or most other outcomes looked at when comparing art therapy to other activities.

\section{Quality of the Evidence}

The studies were limited by many factors that reduced the quality of findings - considered 'very low' with well-known methods for evaluating this. Each study used different types of art therapy. This made it difficult to look at all the results together. One study had a high rate of people not completing the research trial. The studies included small numbers of people, which makes it difficult to be sure how accurate the findings are. This also makes it difficult to know if the effects will be the same in more people. Art therapy is difficult to test for its effects.

More research is needed on this topic. There is not enough information from research trials about the effects of art therapy for people with dementia. This review suggests ways to do this. 
SUMMARY OF FINDINGS

Summary of findings for the main comparison. Summary of findings table: Art therapy compared with active control group activity for people with dementia

\section{Art therapy compared with active control group activity for people with dementia}

Patient or population: people with dementia

Setting: outpatient clinic or day-care and residential settings

Intervention: art therapy

Comparison: active control (calculation or recreational activity)

\begin{tabular}{|c|c|c|c|c|c|c|}
\hline \multirow[t]{2}{*}{ Outcomes } & \multicolumn{2}{|c|}{ Anticipated absolute effects ${ }^{\star}(95 \% \mathrm{Cl})$} & \multirow{2}{*}{$\begin{array}{l}\text { Relative effect } \\
(95 \% \mathrm{Cl})\end{array}$} & \multirow{2}{*}{$\begin{array}{l}\text { № of partici- } \\
\text { pants } \\
\text { (studies) }\end{array}$} & \multirow{2}{*}{$\begin{array}{l}\text { Quality of the } \\
\text { evidence } \\
\text { (GRADE) }\end{array}$} & \multirow[t]{2}{*}{ Comments } \\
\hline & $\begin{array}{l}\text { Risk with active control } \\
\text { (calculation or recreation- } \\
\text { al activity) }\end{array}$ & Risk with art therapy & & & & \\
\hline $\begin{array}{l}\text { Change in MMSE } \\
\text { follow-up: } 12 \text { weeks }\end{array}$ & $\begin{array}{l}\text { The mean change in MMSE } \\
\text { was }+2.1\end{array}$ & $\begin{array}{l}\text { The mean change in MMSE was } \\
-0.2\end{array}$ & - & $\begin{array}{l}39 \\
(1 \mathrm{RCT})\end{array}$ & $\begin{array}{l}\oplus \ominus \ominus \ominus \\
\text { VERY LOW } 12\end{array}$ & - \\
\hline $\begin{array}{l}\text { Change in CSDD } \\
\text { follow-up: } 40 \text { weeks }\end{array}$ & $\begin{array}{l}\text { The mean change in CSDD } \\
\text { was }-0.17\end{array}$ & $\begin{array}{l}\text { The mean change in CSDD was } \\
+5.89\end{array}$ & - & $\begin{array}{l}21 \\
(1 \mathrm{RCT})\end{array}$ & $\begin{array}{l}\oplus \ominus \ominus \ominus \\
\text { VERY LOW a,b,c, }\end{array}$ & - \\
\hline $\begin{array}{l}\text { Change in MCS-8 } \\
\text { follow-up: } 12 \text { weeks }\end{array}$ & $\begin{array}{l}\text { The mean change in MCS- } 8 \\
\text { was }-1.9\end{array}$ & $\begin{array}{l}\text { The mean change in MCS- } 8 \text { was } \\
+2.1\end{array}$ & - & $\begin{array}{l}39 \\
(1 \mathrm{RCT})\end{array}$ & $\begin{array}{l}\oplus \ominus \ominus \ominus \\
\text { VERY LOW a,b }\end{array}$ & - \\
\hline
\end{tabular}

${ }^{\star}$ The risk in the intervention group (and its $95 \%$ confidence interval) is based on the assumed risk in the comparison group and the relative effect of the intervention (and its $95 \% \mathrm{Cl})$.

\section{GRADE Working Group grades of evidence}

High quality: We are very confident that the true effect lies close to that of the estimate of the effect

Moderate quality: We are moderately confident in the effect estimate: The true effect is likely to be close to the estimate of the effect, but there is a possibility that it is substantially different

Low quality: Our confidence in the effect estimate is limited: The true effect may be substantially different from the estimate of the effect

Very low quality: We have very little confidence in the effect estimate: The true effect is likely to be substantially different from the estimate of effect 
bInconsistency - downgraded for methodological heterogeneity and a high degree of inconsistency in the effect on outcomes between studies.

clmprecision - small sample size with results reported for endpoint group sizes of 9 and 12, due to high attrition rates. 


\section{B A C K G R O U N D}

\section{Description of the condition}

Dementia is a progressive clinical syndrome characterised by widespread cognitive impairment. Dementia has a number of different causes and the term encompasses multiple degenerative brain disorders that affect memory, thinking, behaviour, emotion and performance in activities of daily living (Alzheimer's Disease International 2010).

The global prevalence of dementia in 2015 was estimated at 46.8 million, with numbers almost doubling every 20 years, and the estimated prevalence rising to 131.5 million by 2050 (Alzheimer's Disease International 2015). Dementia continues to have a significant socio-economic impact on health and social care systems (Alzheimer's Disease International 2015).

Alzheimer's Disease International has advocated more commissioning and funding of research (Alzheimer's Disease International 2011), including researching the efficacy of pharmacological and non-pharmacological interventions specifically for the early stages of dementia. There is a large body of ongoing research into pharmacological treatments but, traditionally, less has been directed towards non-pharmacological therapies (Van der Steen 2017). Interventions may be broadly categorised as those aimed at modifying disease processes, and targeting the causes and managing manifestations of the disease. Arts-based therapies are generally considered to fall within the last category, as they may help to slow cognitive deterioration, address symptoms related to psychosocially challenging behaviours and improve quality of life.

\section{Description of the intervention}

Art therapy is defined by the British Association of Art Therapists as: "a form of psychotherapy that uses art media as its primary mode of communication. Clients who are referred to an art therapist need not have experience or skill in art. The art therapist is not primarily concerned with making an aesthetic or diagnostic assessment of the client's image. The overall aim of its practitioners is to enable a client to change and grow on a personal level through the use of art materials in a safe and facilitating environment" (BAAT 2013; Ruddy 2005). Our review focuses on studies of art therapy and does not include studies of other arts therapies (dance-movement therapy, drama therapy or music therapy).

Historically, drawings and paintings have been recognised as a useful part of therapeutic processes within psychiatric and psychological specialties (BAAT 2013), and this has been acknowledged within medical and neurology-based scientific audiences (Burton 2009), as well as through media coverage in wider societal contexts (BAAT 2013). In the UK, employment of art therapists by the National Health Service (NHS) began in the 1940 s, and art therapy is now statutorily regulated in the UK (BAAT 2013). It is a legal requirement in the UK that in order to practise professionally, art therapists must graduate from a recognised course and gain registration from the Health and Care Professions Council (BAAT 2013).

\section{How the intervention might work}

There is ongoing discussion about the theoretical base of art therapy. This is complex and may be considered in terms of "dynamic, humanistic, systemic and social understandings of art, therapy, and people" (Huss 2009). Within the literature some authors make a key distinction between art as a treatment (focusing on clinical outcomes) and as an activity (focusing on the creative process itself), whilst others consider art therapy as a creative therapy that offers a holistic approach to a person's condition and illness (Beard 2012a). It is believed that the therapeutic effects relate to the art-making process as well as the interactions comprising the therapist-patient relationship (Edwards 2004). Existing theoretical models may therefore focus on the process and context of art making, or on the use of art as an analytical and projective tool (Huss 2009; Schaverien 1992). A multilayered model has also been suggested, integrating the different theoretical conceptualisations (Huss 2009).

Art therapy involves creative activity and art-making processes targeting cognitive, motor, emotional and interpersonal skills. Art therapy also serves as a non-verbal expressive medium, thereby offering insights into the patient's 'inner world' through a three-way process between the patient, the therapist and art materials (object or image) (BAAT 2013). Experts have suggested potential physiological (neurological) and psychological mechanistic explanations for the effects of visual art interventions. These hypotheses are often inter-related and considered in conjunction with one another.

The general literature cites studies showing that art therapy has measurable subjective benefits in facilitating functioning through reminiscence and rediscovery of obscured abilities; improving quality of life through affirming the individual's sense of self and promoting psychological well-being; and helping reduce perceived problem behaviours associated with dementia (Van der Steen 2017; Waller 2002), as well as indicating the potential for positive objective biomedical treatment outcomes (Alzheimer's Disease International 2011; Beard 2012a). More recently, authors of a study seeking to develop theoretical understanding considered how art gallery-based programmes involving processes of viewing and making art within the physical and social context of the gallery, might impact people with dementia and their caregivers (Camic 2016), discussed the emergence of four components they proposed as critical factors in creating positive emotional and relational effects for participants: intellectual stimulation, the perceived importance of which was considered to be in keeping with the increasing use of cognitive stimulation therapy groups (Spector 2003); the setting of a valued place or environment, away from daily activities or associations with illness; social relationships and interaction including peer support and respite for caregivers; and changed perceptions of dementia, afforded by the shared experience of self-reported positive affect and social inclusion associated with the intervention processes (Camic 2016).

Art therapy involves sensory and intellectual stimulation, and the latter has been considered important in terms of interest, competency and engagement of participants (Camic 2016). There is some emerging evidence for the effects of cognitive interventions such as cognitive stimulation on cognitive function, and hypotheses about the potential effects of cognitive interventions on neuronal processes underlying cognitive impairment, in healthy elderly individuals and in those with mild cognitive impairment and early Alzheimer's disease (Huntley 2015; Bahar-Fuchs 2013; Belleville 2011; Martin 2011). 
A recent neuroimaging study exploring the effects of visual art on the functional neuroanatomy of the brain in post-retirement adults reported that making visual art improved effective interaction between the regions of the brain network being investigated and that these improvements were associated with better scores for psychological resilience, demonstrating links between the basic cognitive neuroscience finding to a clinically-relevant finding with potential therapeutic implications (Bolwerk 2014). Further research is required to examine whether improvements in impaired connectivity in functional brain networks are associated with positive effects on measures of cognition, affect and emotional well-being, behaviour and social functioning in clinical disease states.

The neurological basis of creativity and its role in dementia remains to be elucidated, both in terms of the creative experience of patients and the effects of creative activity on the brain. It has been suggested that there may be links between altered creativity and some psychiatric and neurological diseases, with efforts to explain these in terms of neuropsychology and anatomy, although the nature of these associations remains unclear (Flaherty 2011). Although cognitive function progressively declines during the course of the disease, studies have found that some individuals even with advanced dementia may be able to complete selfportraits when requested (Beard 2012a). Cerebral damage may be reflected in impaired artistic ability, as is often the case in Alzheimer's disease dementia. However, some patients with brain disease display emerging, enduring or enhanced artistry (Flaherty 2011; Schott 2012). This has been highlighted in the temporal variant of fronto-temporal dementia, a relatively rare form of dementia where there is pathological involvement of the anterior temporal lobes whilst the dorsolateral frontal cortex is spared, resulting in adversely-affected language and social skills, but sparing of visual skills, with the acquisition of new artistic skills observed in some patients (Miller 1998). However any inferred implications for treatments such as art therapy are limited by insufficient research evidence (Flaherty 2011).

\section{Why it is important to do this review}

During the last 50 years case studies and qualitative research have assessed the use of art therapy in dementia (BAAT 2013; Burton 2009); however, our review aims to summarise higherquality evidence of its effects from randomised controlled trials. A systematic review of evidence for the effects of art therapy in the care of people with dementia is required to inform future clinical applications and research directions regarding this form of therapy.

The benefits of pharmacological treatments are limited in the longterm management of many features of dementia (Van der Steen 2017). Non-pharmacological interventions are considered to be an important adjunctive approach. Non-pharmacological therapies for dementia that have been suggested previously include cognitive stimulation and reality orientation, reminiscence therapy, psychological treatments, including cognitive behavioural therapy, psychosocial interventions, physical exercise, caregiver-focused interventions, drama therapy and music therapy (Alzheimer's Disease International 2011; Beard 2012a; Van der Steen 2017).

The advantages of non-pharmacological interventions include the lack of typical drug-associated adverse effects and potential cost-saving benefits, including delayed institutionalisation and enhanced quality of life (Alzheimer's Disease International 2011).
These interventions may serve as an appropriate adjunct to pharmacological therapies, or may offer a suitable alternative for the mainstay of therapy in those individuals who do not meet evidence-based criteria for acetylcholinesterase inhibitor drugs (Alzheimer's Disease International 2011).

In a broader sense, this review of art therapy contributes to the existing body of evidence for non-pharmacological groupbased activities and psychological therapies for people with dementia. It is possible there may be some overlap in nonpharmacological group and activity-based interventions involving similar components to those hypothesised to be key to the mechanisms of action of art-based therapies, including the group setting and social interaction, engaging in enjoyable activities, and providing stimulation for cognitive processes (BAAT 2013; Bolwerk 2014; Camic 2016; Woods 2012). This review of the efficacy of art therapy for people with dementia is important to add to the existing evidence base for cognitive and psychological interventions as well as group-based creative activities for people with dementia.

BAAT identifies art therapy as a form of psychotherapy and National Institute for Health and Care Excellence (NICE) guidelines currently recommend psychological interventions for people with dementia with co-morbid emotional disorders such as depression or anxiety or both, including a range of tailored interventions such as reminiscence therapy and multisensory stimulation (NICE 2006). NICE guidelines recommend therapeutic use of creative arts based activities tailored to individual preferences, skills and abilities, including music and dancing, for people with dementia with comorbid agitation (NICE 2006).

\section{O B JECTIVES}

To review the effects of art therapy as an adjunctive treatment for dementia compared with standard care and other nonpharmacological interventions.

\section{METHODS}

\section{Criteria for considering studies for this review}

\section{Types of studies}

Randomised controlled trials (RCTs) in which art therapy was used as an intervention for people with any form of dementia, compared with standard care or other psychosocial interventions for dementia.

\section{Types of participants}

We included studies in which participants had a formal (clinicianconfirmed) diagnosis of any form of dementia. We included participants resident in the community or in any form of institutional care and with all severities of dementia.

\section{Types of interventions}

The experimental intervention of art therapy, administered to a group or individually. We considered a minimum of five sessions to constitute a therapeutic intervention. We used the aforementioned British Association of Art Therapists' definition of art therapy as a gold standard for inclusion (BAAT 2013). We did not include studies that used multi-modal interventions in which it was not possible to distinguish the specific effects of the art therapy component. 
Acceptable control interventions included no treatment, usual care, or any other form of non-pharmacological intervention for dementia.

\section{Types of outcome measures}

\section{Primary outcomes}

- Cognition: includes the following cognitive domains: attention/ orientation, memory, fluency, language skills (speech content and fluency) and visuospatial perception

- Affect and emotional well-being (where 'affect' is considered the fluctuating changes in emotion or feelings)

- Social functioning

- Behaviour

- Adverse effects

We accepted all validated cognitive, behavioural and psychological scales, and global impression measures reported by authors of the primary studies and deemed appropriate by the review authors.

\section{Secondary outcomes}

- Death

- Quality of life

- Economic outcomes

\section{Search methods for identification of studies}

\section{Electronic searches}

We searched the Cochrane Dementia and Cognitive Improvement Group's Specialised Register, on 12 May 2014, 20 March 2015, 15 January 2016, 4 November 2016, and 4 October 2017. The search terms we used included: art, artistic, artist, artwork, draw, drawing, paint, painting, sketch, sketching, doodle.

ALOIS is maintained by the Cochrane Dementia and Cognitive Improvement Group's Information Specialists and contains dementia and cognitive-improvement studies identified from:

1. Monthly searches of a number of major healthcare databases: MEDLINE, Embase, CINAHL, PsycINFO and LILACS

2. Monthly searches of a number of trial registers: metaRegister of Controlled Trials; Umin Japan Trial Register; WHO portal (which covers ClinicalTrials.gov; ISRCTN; Chinese Clinical Trials Register; German Clinical Trials Register; Iranian Registry of Clinical Trials; and the Netherlands National Trials Register, plus others

3. Quarterly searches of the Cochrane Library's Central Register of Controlled trials (CENTRAL)

4. Six-monthly searches of a number of grey literature sources: ISI Web of Knowledge Conference Proceedings; Index to Theses; Australasian Digital Theses

We ran additional separate searches in many of the above sources to ensure that we retrieved the most up-to-date results. The search strategy used for the retrieval of reports of trials from MEDLINE (via the Ovid SP platform) can be seen in Appendix 1.

\section{Searching other resources}

\section{Handsearching}

We handsearched the grey literature, such as conference proceedings, and searched the reference lists of all the included studies as well as other potentially relevant trials identified through the above search strategy. We did not apply any language restrictions.

\section{Personal contact}

We contacted authors of relevant reviews or studies to enquire about other sources of relevant information. We had already contacted Val Huet, the Chief Executive Officer for the British Association of Art Therapists as part of the review proposal process. We contacted Professor Diane Waller, a co-author of a relevant study.

\section{Data collection and analysis}

\section{Selection of studies}

Two review authors independently assessed all the studies identified through the search strategy for eligibility, by checking the title or abstract or both. If there was any doubt about an article's relevance, we assessed the full-text article. Where necessary, we resolved any disagreement through discussion or, if required, we consulted a third party. Where necessary, we contacted authors of the original reports to provide further information.

\section{Data extraction and management}

For eligible studies, two review authors extracted and crosschecked outcome data independently. We used a proforma that we had designed for data collection.

\section{Assessment of risk of bias in included studies}

We used the Cochrane 'Risk of bias' tool, a domain-based evaluation (Higgins 2011). Two review authors independently assessed risks of bias for included studies using the criteria outlined in the Cochrane Handbook for Systematic Reviews of Interventions (Higgins 2011). We assessed the following elements of each included study: sequence generation, allocation concealment, blinding of participants, personnel and outcome assessors, incomplete outcome data, selective reporting bias and other potential sources and risks of bias (Higgins 2011).

\section{Measures of treatment effect}

\section{Dichotomous data}

We reported data for MCS-8 (a quality-of-life measure) as an odds ratio (OR).

\section{Continuous data}

Most outcome measures were continuous data reported as the mean change in each group where the studies published their results as mean values for each group at baseline and postintervention, allowing the absolute difference between the mean value to be calculated as an estimate of the amount by which the intervention or control changed the outcome on average. For most outcome measures we did not report the standard deviation of the changes, confidence intervals and $\mathrm{P}$ values for differences between intervention and control groups. We reported data for Mini-Mental State Examination (MMSE: a cognitive-impairment measure) in 
terms of $\mathrm{P}$ values for differences between intervention and control groups.

\section{Dealing with missing data}

For included studies, we noted levels of attrition. We discuss the potential impact of missing data on the findings of the review.

\section{RES U L T S}

\section{Description of studies}

See: Characteristics of included studies and Characteristics of excluded studies

We identified 922 results after de-duplication for assessment. Two studies met the criteria for inclusion. The studies varied in participant characteristics, length of individual art therapy sessions, number of sessions and duration, nature of the activities defined as art therapy, the nature of the control condition, and outcome measures. We consider these factors below in turn.

\section{(1) Study settings}

Hattori 2011: Hospital (outpatient basis - psychiatry or memory clinic) in Obu, Aichi, Japan.

Rusted 2006: Day centre or residential facility in East Sussex, UK.

\section{(2) Participant characteristics}

Hattori 2011: The intervention group was composed of 20 participants ( 11 women) with a mean age of $75.3 \pm 5.3$ years and baseline MMSE $24.6 \pm 3.4$. The control group was composed of 19 participants ( 10 women) with an average age of $73.3 \pm 6.3$ years and baseline MMSE $22.3 \pm 2.7$. Four of 43 originally recruited participants dropped out due to physical diseases. We present results for the 39 participants completing the 12-week study: intervention group $(n=$ $20)$ and control group $(n=19)$.

The study excluded patients on donepezil for less than six months, those with an MMSE score of 19 and under or an MMSE score 25 or more and not fulfilling the inclusion criteria, and those with primary symptoms related to speech and impaired executive function, without findings characteristic of Alzheimer's disease. Participants were recruited from the outpatient clinic of the Department of Psychiatry or Memory Clinic, National Center for Geriatrics and Gerontology.

Rusted 2006: The study included 31 female participants with an average age of 84.05 years (range 74 to 92) and 14 male participants with an average age of 80.33 years (range 67 to 92). Twenty-four of 45 originally-recruited participants dropped out due to death, ill health, changes in care provision, transport or changes in residence beyond the participants' control. Results are presented for the 21 participants completing the 40-week study: intervention group ( $n=$ 9) and control group $(n=12)$.

In this study different types of dementia diagnosis amongst participants were as follows: Alzheimer type ( $n=18 ; 11$ women), multi-infarct dementia ( $n=19 ; 13$ women), unspecified dementia ( $\mathrm{n}=8 ; 7$ females). Different severities of dementia diagnosis amongst participants were as follows: mild $(n=4)$, moderate $(n=8)$, severe $(n=33)$. At baseline there was an imbalance in depressive symptomatology, with higher mean CSDD scores in the art therapy group compared with the activity group $(F(1,19)=8.65, P$
$<0.01)$. The study excluded people with psychiatric co-morbidities. Participants were recruited from those attending at day care and care-home residents.

\section{(3) Length and duration of sessions and nature of activities}

Hattori 2011: Groups of approximately five participants per session met for weekly 45-minute sessions, for 12 weeks. The art therapy intervention involved a combination of several techniques. The main task was to colour with pastel crayons or water-based paint pre-prepared abstract patterns. Additional tasks were to colour line drawings of familiar objects such as flowers, children and fish or draw pictures based on memories or favourite seasons. The intervention was delivered by an industrial designer and artist and three speech therapists. Participants were also instructed to carry out tasks within their capability for approximately 15 minutes daily. In the control group, the task involved performing simple calculations (additions and multiplications of 1- and 2-digit numbers). In both groups, participants were accompanied by their family members.

Rusted 2006: Groups of up to six participants met for weekly onehour sessions for 40 weeks. The art therapy intervention involved a group interactive, psychodynamic approach with a variety of art materials presented for use. The intervention was delivered by an art therapist or occupational therapist and an assistant, who had attended induction meetings where the research protocol was outlined and handbooks on this were provided. Art therapists had supervision sessions with the senior art therapist at least fortnightly. Assistants worked under the guidance of the art therapists and had supervision with the psychologist regularly. In the control group, a selection of recreational activities were used, excluding any formal occupational therapy methods or any form of art and craft work.

\section{(4) Outcome measures}

Hattori 2011: MMSE. Increase in score represents improvement. Wechsler Memory Scale-Revised (WMS-R) sub-scale evaluating logical memory (WMS-log). Increase in score means improvement. Geriatric Depression Scale (GDS). Reduction in score means improvement. Apathy Scale (Japanese version). Reduction in score means improvement. Physical Component Summary (PCS-8). Increase in score means improvement. Mental Component Summary (MCS-8). Increase in score represents improvement. Dementia Behaviour Disturbance Scale (DBD). Reduction in score means improvement. Barthel Index. Increase in score means improvement. Zarit Caregiver Burden Interview (CBI). Reduction in score means improvement. Measurements were recorded at baseline and at 12 weeks.

Rusted 2006: Cornell Scale for Depression in Dementia (CSDD). Reduction in score represents improvement. The Multi Observational Scale for the Elderly (MOSES). Reduction in score means improvement. MMSE. Increase in score means improvement. Rivermead Behavioural Memory Test (RBMT) subtest to assess short-term memory (RBM-STM). Reduction in score means improvement. Cornell Scale for Depression in Dementia (CSDD). Reduction in score represents improvement. Tests of Everyday Attention (TEA). Benton Fluency Task (BFT). Measurements were recorded at baseline, 10 weeks, 20 weeks, 40 weeks and at follow-up (44 weeks and 56 weeks). Within-session measures were also used. However this review considers only preand post-intervention outcome measures. 


\section{Risk of bias in included studies}

See: Characteristics of included studies for 'Risk of bias' tables.

\section{Effects of interventions}

See: Summary of findings for the main comparison Summary of findings table: Art therapy compared with active control group activity for people with dementia

See: Characteristics of included studies for Summary of findings for the main comparison.

It was not possible to obtain adequate data to pool in metaanalyses. Neither study defined outcome measures as primary or secondary endpoints. There were no clear differences between groups reported for the outcome measures in question. We considered the quality of the evidence to be 'very low' using a GRADE rating (see: Summary of findings for the main comparison). The findings reported should be interpreted with caution in view of the limitations identified.

Hattori 2011: At 12 weeks, cognition (MMSE) favoured the calculation group compared with the art-therapy group, whereas the mental component of health-related quality of life (MCS-8 subscale of SF-8) favoured art therapy compared with learning therapy performing simple calculations. MMSE score improved in the calculation (control) group, compared with the art-therapy (intervention) group.

Rusted 2006: At 40 weeks, anxious and depressive symptomatology (CSDD and MOSES) increased in the art-therapy group, compared with the control group, but the art-therapy group were more depressed at baseline compared with the control group. No changes were reported in measures of cognition, memory or attention.

We summarised reported effects of interventions from the two studies included for all prespecified primary and secondary outcome measures as follows. No confidence intervals were reported.

\section{Primary outcomes}

\section{Cognition}

Hattori 2011: The control group mean MMSE score increased compared with the intervention group mean MMSE score which decreased: intervention $(-0.2)$ versus control (+2.1).

Rusted 2006: No differences between groups were reported for MMSE, the Rivermead Behavioural Memory Test, Tests of Everyday Attention and the Benton Fluency Task.

\section{Affect and emotional well-being}

Hattori 2011: No differences were reported in Apathy Scale or GDS when comparing the two groups.

Rusted 2006: The intervention group showed worsening from baseline in mean CSDD $(P<0.05)$. The intervention group showed worsening from baseline in mean MOSES score for 'depressed/ anxious mood' $(\mathrm{P}<0.05)$.

\section{Social functioning and behaviour}

Hattori 2011: No difference was reported in DBD when comparing the two groups.

\section{Adverse effects}

Rusted 2006: No clear differences were reported in adverse effects when comparing the groups. The intervention group were more depressed at baseline, so were not comparable to the control group.

\section{Secondary outcomes}

Death

Hattori 2011: No information was reported.

Rusted 2006: Ten deaths were reported as unrelated to the trial and no information was reported on how many were in each group.

\section{Quality of life}

Hattori 2011: Percentage showing at least 10\% improvement from baseline in MCS- 8 was compared between groups by $\mathrm{Chi}^{2}$ test and favoured the intervention group $(P=0.038$, odds ratio 5.54). No difference was reported in Zarit CBI when comparing the two whole groups.

\section{Economic outcomes}

No economic outcomes were reported.

\section{DISCUSSION}

\section{Summary of main results}

We found no clear differences between intervention and control groups, and we rated the quality of the evidence 'very low' using a GRADE rating (see: Summary of findings for the main comparison).

\section{Overall completeness and applicability of evidence}

There is limited information available on the effectiveness of art therapy. Searching the literature revealed only two relatively small randomised controlled trials, with results reported for a total of 60 participants, that were suitable for inclusion.

Overall, regarding the nature of the interventions classified here as art therapy, more detailed descriptions of the interventions could be provided (this may be difficult to achieve where a standardised prescribed protocol is not used) and consideration of ways to monitor therapist adherence to the intervention protocol. The selection of outcome measures appears appropriate, reflecting an evaluation of change in key domains as well as one study including broader outcome measures to evaluate potential impact on caregivers. However neither study defined primary and secondary endpoints.

We assessed both studies for the quality of the description of interventions, using the Template for Intervention Description and Replication (TIDieR) checklist and guide (Hoffmann 2014). This is summarised in Table 1. A common and recurring theme was the need for clarity about the underlying theory or rationale or both for study design decisions. 


\section{Quality of the evidence}

(See: Summary of findings for the main comparison and Characteristics of included studies 'Risk of bias' tables)

We used the GRADE approach in interpreting findings (Langendam 2013; Schünemann 2013).

Overall we judged the evidence for our main outcomes to be of 'very low' quality, using the GRADE approach. There were serious limitations in the data available on the outcomes of interest. We judged the studies to be at very serious risk of bias in terms of performance bias and attrition bias. In relation to the findings, there are important methodological issues to be considered, including lack of clarity in the method of randomisation, lack of allocation concealment and blinding (it is unclear in one study whether the same personnel delivered the art therapy and control interventions as well as assessing outcome measures), and attrition rates. The individual outcomes examined would be downgraded as a result.

\section{Potential biases in the review process}

We used standard procedures, as specified, to identify all relevant studies. We tried, in line with our protocol, to ensure that we found all relevant published and accessible studies, including contacting authors of included and excluded studies. We made decisions about analysis of data based on our assessment of the quality of the studies and the data available. For both studies, it was unclear whether re-analysis was possible or appropriate with the information available.

\section{Agreements and disagreements with other studies or reviews}

Overall, the evidence about the effectiveness of art therapy for people with dementia remains limited and inconclusive. Existing studies vary in participant characteristics, length of individual arttherapy sessions, number of sessions and duration, nature of the activities defined as art therapy, the nature of the control condition, and outcome measures.

\section{AUTHORS' CONCLUSIONS}

\section{Implications for practice}

There is insufficient evidence from randomised controlled trials to draw any reliable conclusions about the efficacy of art therapy for people with dementia.

Given the popularity of art therapy with therapists and clients, and in view of its ongoing clinical use, further and better evaluation should be pursued.

\section{Implications for research}

There is a clear need for more RCTs of art therapy for people with dementia. Future research on art therapy should seek to address the methodological limitations identified in existing studies and should aim to establish any effects directly attributable to art therapy as a discrete single-modality intervention. More clearlydefined treatment protocols are required to identify the key therapeutic elements and evaluate effects of different approaches. Future research should also seek to evaluate a wider range of outcomes for patients, caregivers and staff. Any negative effects must be assessed and reported to identify whether there are any harmful effects.

There are a number of challenges faced by researchers in conducting RCTs investigating the efficacy of art therapy for people with dementia. It is important to acknowledge specific challenges in adapting complex interventions such as art therapy for assessment in RCTs. The Medical Research Council (MRC) framework provides guidance (Craig 2008). Challenges for researchers include consideration of the variation in art therapy models and contextual, therapist and participant factors. Pragmatic trials of complex interventions might involve standardisation of function rather than form (Patterson 2015). This suggests that when designing pragmatic trials of complex interventions such as art therapy, for the internal and external validity of the test, interventions are standardised by function with flexibility in form, including content or delivery of the intervention. For example, it may be more effective to tailor the delivery of art therapy to the context, i.e. to the individual and their circumstances, rather than always using exactly the same art materials and approaches for 'treatment fidelity' (Hawe 2008).

The identified limitations in existing studies need to be addressed. When designing studies, researchers should consider guidance from the MRC Framework for the Development and Evaluation of RCTs for Complex Interventions to Improve Health (Craig 2008) and the TIDieR checklist (Hoffmann 2014). Investigators should aim to test clearly-defined hypotheses. Research should also address cost effectiveness and wider deliverability of the intervention. Feasibility studies should be conducted to estimate important parameters including sample size and recruitment factors. Formal supervision of intervention and control group facilitators might provide insights into the effects on staff and issues with patient adherence. The influence of contextual factors on outcomes might be better understood through process evaluation (Craig 2008). Study results, including outcome data and recommendations arising from the findings, should be reported clearly.

Potential treatment effect modifiers identified in the two studies included in this review include experience and expertise of the art therapist, group size and composition, and environmental setting. This is based on factors suggested to be important in mechanisms of art therapy, which include the therapeutic relationship established with the therapist, the group setting, social interactions and relationships between group members, and perceptions of the environment. The art-therapy intervention was delivered by individuals from a wide range of backgrounds with varying levels of experience and expertise. The group setting varied, with carers being present in one study. The environment differed between the two studies (community setting versus hospital setting). We identified a further potential treatment effect modifier as the substantial heterogeneity in type and severity of dementia.

Future systematic reviews should incorporate subgroup analyses where possible. These might cover evaluating art therapy for people with dementia of different forms and severities, group versus individual therapy, therapy in the community versus in institutional care, differences between self-reported and observerrated outcomes, and differences between cluster-randomised trials and non-cluster-randomised trials. It may be difficult to focus the population of interest (in terms of clinical profile) unless there are more high-quality RCTs. The PICO mnemonic (population, intervention, comparison, and outcomes) provides a structured 
format to consider each element when formulating a systematic review question. This is applied here for future studies in Table 2.

The above conclusions offer an outline of key challenges and considerations for future research, based on limitations identified in the existing evidence base.

\section{ACKNOWLEDGEMENTS}

As part of the pre-publication editorial process, the protocol and review underwent editorial and external peer review. Anna Noel-Storr, the Group's Information Specialist was involved in developing the search strategy. We also contacted Val Huet, the Chief Executive Officer for the British Association of Art Therapists, as part of the review proposal process. 


\section{R E F E R E N C E S}

\section{References to studies included in this review}

Hattori 2011 \{published data only\}

Hattori H, Hattori C, Hokao C, Mizushima K, Mase T. Controlled study on the cognitive and psychological effect of coloring and drawing in mild Alzheimer's disease patients. Geriatrics \& Gerontology International 2011;11(4):431-7.

\section{Rusted 2006 \{published data only\}}

Rusted J, Sheppard L, Waller D. A multi-centre randomised control group trial on the use of art therapy for older people with dementia. Group Analysis 2006;39(4):517-36.

\section{References to studies excluded from this review}

Camic 2014 \{published data only\}

Camic PM, Tischler V, Pearman CH. Viewing and making art together: a multi-session art-gallery-based intervention for people with dementia and their carers. Aging \& Mental Health 2014;18(2):161-8.

\section{Fostinelli 2016 \{published data only\}}

Fostinelli S, Uggeri G, Facchinetti M, Benussi L, Ghidoni R, Di Giovanni G, et al. Efficacy of a group visual art therapy with frontotemporal dementia patients: a pilot study. 10th International Conference on Frontotemporal Dementias Munich/Germany. 2016.

\section{Han 2010 \{published data only\}}

Han P, Kwan M, Chen D, Yuseff SZ, Chionh HL, Goh J, et al. A controlled naturalistic study on a weekly music therapy and activity program on disruptive and depressive behaviors in dementia. Dementia and Cognitive Disorders 2010;30(6):540-6.

\section{Jensen 1997 \{published data only\}}

Jensen SM. Multiple pathways to self: a multisensory art experience. Art Therapy: Journal of the American Art Therapy Association 1997;14(3):178-86.

\section{Kong 2015 \{published data only\}}

Kong M. The effects of group art therapy on the cognitive functions of the elderly with dementia. Korean Journal of Art Therapy 2015;6:1829-53.

\section{MacPherson 2009 \{published data only\}}

MacPherson S, Bird M, Anderson K, Davis T, Blair A. An art gallery access programme for people with dementia: 'you do it for the moment'. Aging \& Mental Health 2009;13(5):744-52.

\section{NHS 2012 \{published data only\}}

National Opera Company \& NHS Trust. Arts project offers trip down memory lane. Nursing Older People 2012;24(6):4.

\section{Odell-Miller 2006 \{published data only\}}

Odell-Miller $\mathrm{H}$, Hughes $\mathrm{P}$, Westacott $\mathrm{M}$. An investigation into the effectiveness of the arts therapies for adults with continuing mental health problems. Psychotherapy Research 2006;16(1):122-39.
Rusted 2002 \{published data only\}

Rusted J, Sheppard L, Waller D. Art therapy improves social functioning and communication in people with dementia. 8th International Conference on Alzheimer's Disease and Related Disorders; 20 July 2002; Stockholm, Sweden. 2002:Abstract No: 197.

\section{Rylatt 2012 \{published data only\}}

Rylatt $P$. The benefits of creative therapy for people with dementia. Nursing Standard 2012;26(33):42-7.

Safar 2011 \{published data only\}

Safar LT, Press DZ. Art and the brain: effects of dementia on art production in art therapy. Art Therapy: Journal of the American Art Therapy Association 2011;28(3):96-103.

Seifert 2003 \{published data only\}

Seifert LS, Baker MK. Art and Alzheimer-type dementia: a longitudinal study. Clinical Gerontologist 2003;26(1/2):3-15.

Stallings 2010 \{published data only\}

Stallings JW. Collage as a therapeutic modality for reminiscence in patients with dementia. Art Therapy: Journal of the American Art Therapy Association 2010;27(3):136-40.

Ullan 2013 \{published data only\}

Ullan AM, Belver MH, Badia M, Moreno C, Garrido E, GomezIsla J, et al. Contributions of an artistic educational program for older people with early dementia: an exploratory qualitative study. Dementia (Basel, Switzerland) 2013;12(4):425-46.

Walsh 2011 \{published data only\}

Walsh SM, Lamet AR, Lindgren CL, Rillstone P, Little DJ, Steffey CM. Art in Alzheimer's care: promoting well-being in people with late-stage Alzheimer's disease. Rehabilitation Nursing 2011;36(2):66-72.

\section{Additional references}

\section{Altman 2005}

Altman DG, Bland JM. Treatment allocation by minimisation. BMJ 2005;330(7495):843.

\section{Alzheimer's Disease International 2010}

Alzheimer's Disease International. World Alzheimer Report 2010: The Global Economic Impact of Dementia. www.alz.co.uk/ research/world-report-2010 (accessed 20 July 2018).

\section{Alzheimer's Disease International 2011}

Alzheimer's Disease International. World Alzheimer Report 2011: The Benefits of Early Diagnosis and Intervention. www.alz.co.uk/research/world-report-2011 (accesed 20 July 2018).

\section{Alzheimer's Disease International 2015}

Alzheimer's Disease International. World Alzheimer Report 2015: The Global Impact of Dementia. An Analysis of Prevalence, 
Incidence, Cost and Trends. www.alz.co.uk/research/worldreport-2015 (accessed 20 July 2018).

\section{BAAT 2013}

British Association of Art Therapists website. www.baat.org (accessed 23 December 2013).

\section{Bahar-Fuchs 2013}

Bahar-Fuchs A, Clare L, Woods B. Cognitive training and cognitive rehabilitation for mild to moderate Alzheimer's disease and vascular dementia. Cochrane Database of Systematic Reviews 2013, Issue 6. [DOI: 10.1002/14651858.CD003260.pub2]

\section{Belleville 2011}

Belleville S, Clement F, Mellah S, Gilbert B, Fontaine F, Gauthier S. Training-related brain plasticity in subjects at risk of developing Alzheimer's disease. Brain 2011;134(6):1623-34

\section{Bolwerk 2014}

Bolwerk A, Mack-Andrick J, Lang FR, Dörfler A, Maihofner C. How art changes your brain: differential effects of visual art production and cognitive art evaluation on functional brain connectivity. PLOS ONE 2014;9(7):e101035. [DOI: 10.1371/ journal.pone.0101035]

\section{Burton 2009}

Burton A. Bringing arts-based therapies in from the scientific cold. Lancet Neurology 2009;8(9):784-5.

\section{Camic 2016}

Camic PM, Baker EL, Tischler V. Theorizing how art gallery interventions impact people with dementia and their caregivers. Gerontologist 2016;56(6):1033-41.

\section{Craig 2008}

Craig P, Dieppe P, Macintyre S, Michie S, Nazareth I, Petticrew M, et al. Developing and evaluating complex interventions: the new Medical Research Council guidance. BMJ 2008;337:a1655.

\section{Edwards 2004}

Edwards D. Art Therapy. Sage, 2004.

\section{Flaherty 2011}

Flaherty AW. Brain illness and creativity: mechanisms and treatment risks. Canadian Journal of Psychiatry 2011;56(3):132-43.

\section{Hawe 2008}

Hawe P, Shiell A, Riley T. Important considerations for standardizing complex interventions in public health. Journal of Advanced Nursing 2008;62:267.

\section{Higgins 2011}

Higgins JP, Altman DG, Sterne JA, editor(s). Chapter 8: Assessing risk of bias in included studies. In: Higgins JP, Green S, editor(s). Cochrane Handbook for Systematic Reviews of Interventions 5.1.0 [updated March 2011]. The Cochrane Collaboration, 2011. Available from www.cochrane-handbook.org.

\section{Hoffmann 2014}

Hoffmann TC, Glasziou PP, Boutron I, Milne R, Perera R, Moher D, et al. Better reporting of interventions: template for intervention description and replication (TIDieR) checklist and guide. BMJ 2014;348:g1687.

\section{Huntley 2015}

Huntley JD, Gould RL, Liu K, Smith M, Howard RJ. Do cognitive interventions improve general cognition in dementia? A metaanalysis and meta-regression. BMJ Open 2015;5:e005247.

\section{Huss 2009}

Huss E. 'A coat of many colors:' Towards an integrative multilayered model of art therapy. Arts in Psychotherapy 2009;36:154-60.

\section{Langendam 2013}

Langendam MW, Akl EA, Dahm P, Glasziou P, Guyatt G, Schünemann HJ. Assessing and presenting summaries of evidence in Cochrane Reviews. Systematic Reviews 2013;23(2):81.

\section{Martin 2011}

Martin M, Clare L, Altgassen AM, Cameron MH, Zehnder F. Cognition-based interventions for healthy older people and people with mild cognitive impairment. Cochrane Database of Systematic Reviews 2011, Issue 1. [DOI: 10.1002/14651858.CD006220.pub2]

\section{Miller 1998}

Miller BL, Cummings J, Mishkin F, Boone K, Prince F, Ponton M. Emergence of artistic talent in frontotemporal dementia. Neurology 1998;51(4):978-82.

\section{NICE 2006}

NICE. Dementia: supporting people with dementia and their carers in health and social care. NICE Clinical Guideline 422006.

\section{Patterson 2015}

Patterson S, Waller D, Killaspy H, Crawford M. Riding the wake: detailing the art therapy delivered in the MATISSE study. International Journal of Art Therapy 2015;20(1):28-38.

\section{Ruddy 2005}

Ruddy R, Milnes D. Art therapy for schizophrenia or schizophrenia-like illnesses. Cochrane Database of Systematic Reviews 2005, Issue 4. [DOI: 10.1002/14651858.CD003728.pub2]

\section{Schaverien 1992}

Schaverien J. Transference and countertransference. The Revealing Image: Analytical Art Psychotherapy in Theory and Practice. London, New York: Tavistock/Routledge, 1992.

\section{Schott 2012}

Schott GD. Pictures as a neurological tool: lessons from enhanced and emergent artistry in brain disease. Brain 2012;135(6):1947-63.

\section{Schünemann 2013}

Schünemann H, Brozek J, Guyatt G, Oxman A, editors. GRADE Handbook for Grading Quality of Evidence and Strength of 
Recommendations. www.guidelinedevelopment.org/handbook October 2013 (accessed 20 July 2018).

\section{Spector 2003}

Spector A, Thorgrimsen L, Woods B, Royan L, Davies S, Butterworth $M$, et al. Efficacy of an evidence-based cognitive stimulation therapy programme for people with dementia: randomised controlled trial. British Journal of Psychiatry 2003;183:248-54.

\section{Van der Steen 2017}

Van der Steen JT, Van Soest-Poortvliet MC, Van der Wouden JC, Bruinsma MS, Scholten RJ, Vink AC. Music-based therapeutic interventions for people with dementia. Cochrane Database of Systematic Reviews 2017, Issue 5. [DOI: 10.1002/14651858.CD003477.pub3]

\section{CHARACTERISTICS OF STUDIES}

Characteristics of included studies [ordered by study ID]

\section{Waller 2002}

Waller D. Evaluating the use of art therapy for older people with dementia: a control group study. In: D Waller editor(s). Arts Therapies and Progressive Illness: Nameless Dread. Hove and New York: Brunner-Routledge, 2002.

\section{Woods 2012}

Woods B, Aguirre E, Spector AE, Orrell M. Cognitive stimulation to improve cognitive functioning in people with dementia. Cochrane Database of Systematic Reviews 2012, Issue 2. [DOI: 10.1002/14651858.CD005562.pub2]

\section{References to other published versions of this review Deshmukh 2014}

Deshmukh SR, Holmes J, Cardno A. Art therapy for people with dementia (Protocol). Cochrane Database of Systematic Reviews 2014, Issue 4. [DOI: 10.1002/14651858.CD011073]

\section{Hattori 2011}

\begin{tabular}{|c|c|}
\hline Methods & $\begin{array}{l}\text { Randomised controlled trial } \\
\text { The study start and end dates were not stated }\end{array}$ \\
\hline Participants & $\begin{array}{l}43 \text { participants with a formal diagnosis of Alzheimer's disease, MMSE } 20 \text { or higher and characteristic } \\
\text { neuroimaging findings } \\
\text { Attendance at hospital (outpatient clinic) and accompanied by family } \\
\text { Data reported for } 39 \text { participants }\end{array}$ \\
\hline Interventions & $\begin{array}{l}\text { Art therapy (experimental) group } \\
\text { Learning therapy performing simple calculations (control) group } \\
\text { Treatment is } 45 \text { minute sessions, weekly, for } 12 \text { weeks }\end{array}$ \\
\hline
\end{tabular}

Outcomes
Affect and emotional well-being: mood and vitality (GDS and Apathy Scale)
Social functioning and Behaviour: behavioural abnormalities (DBD), sense of burden of the partici-
pants' families (Barthel Index and Zarit CBI)
Quality of life: SF-8

Notes

Study funding sources: comprehensive Research on Aging and Health (as part of Health and Labor Sciences Research Grants 2006)

\section{Risk of bias}

\section{Bias \\ Authors' judgement Support for judgement}

Random sequence genera- Unclear risk tion (selection bias)
Minimisation was reportedly used, which may be acceptable as a valid parallel or alternative approach to stratified randomisation that aims to ensure balance between groups across multiple factors, even in small samples (Altman 
2005). However it is arguable that minimisation allocation lacks proper randomisation and may only prove valid in certain experimental contexts. Further details of the method of allocation used are not stated in this study, so the judgement of risk of selection bias remains unclear.

Allocation concealment $\quad$ Unclear risk Incompletely defined or insufficient information provided by the study authors
(selection bias)
(selection bias)

High risk 3 of the 4 therapists delivered both interventions

Blinding of participants and personnel (perfor-

mance bias)

All outcomes

Blinding of outcome as-

Unclear risk

Not stated who carried out the assessments but is likely to be the investigators sessment (detection bias)

All outcomes

$\begin{array}{ll}\begin{array}{l}\text { Incomplete outcome data } \\ \text { (attrition bias) }\end{array} & \text { High risk } \\ \text { All outcomes } & \begin{array}{l}\text { Dropouts not included in the analysis (4 participants did not complete the } \\ \text { study) }\end{array}\end{array}$

\begin{tabular}{|c|c|c|}
\hline Other bias & Unclear risk & $\begin{array}{l}\text { Both groups are compared before and after but not head-to-head, apart from } \\
\text { responders showing a } 10 \% \text { or more improvement in individual outcome mea- } \\
\text { sures. Numbers are not stated. }\end{array}$ \\
\hline
\end{tabular}

Rusted 2006

\begin{tabular}{ll} 
Methods & Randomised controlled trial (parallel, individual) \\
& The study was completed in 2002 \\
\hline
\end{tabular}

Participants 45 people with a formal diagnosis of dementia (diagnosed by consultant psychogeriatrician)
Attendance at day care or residential facility
Data reported for 21 participants

\begin{tabular}{ll}
\hline Interventions & Art therapy (experimental) group \\
& Activity (control) group \\
& Treatment is weekly 1-hour sessions for 40 weeks \\
\hline Outcomes & $\begin{array}{l}\text { Cognition: Cognitive impairment (MMSE), Short term memory (RBMT), Auditory and visual attention } \\
\text { (TEA), Verbal fluency (BFT) } \\
\text { Affect and emotional well-being: depressive symptomatology (CSDD) } \\
\text { All listed primary outcomes: cognition, affect and emotional well-being, social functioning and behav- } \\
\text { iour: self-care functioning, disorientated behaviour, depressed/anxious mood, irritable behaviour, so- } \\
\text { ciability and withdrawn behaviour (MOSES) }\end{array}$
\end{tabular}

Notes Study funding sources: PPP (Health) Foundation

\section{Risk of bias}

Authors' judgement Support for judgement 
Rusted 2006 (Continued)

Random sequence genera- Unclear risk Quote: "Participants were randomly assigned... based on participants' ID numtion (selection bias)

Allocation concealment Unclear risk

Incompletely defined or insufficient information provided by the study authors

(selection bias)

Blinding of participants High risk

and personnel (perfor-

Not reported and the intervention in question is difficult to blind participants

mance bias)

All outcomes

\begin{tabular}{lll}
$\begin{array}{l}\text { Blinding of outcome as- } \\
\text { sessment (detection bias) } \\
\text { All outcomes }\end{array}$ & Unclear risk & Not reported \\
\hline $\begin{array}{l}\text { Incomplete outcome data } \\
\text { (attrition bias) } \\
\text { All outcomes }\end{array}$ & High risk & $\begin{array}{l}\text { High dropout rate is not accounted for in the analysis }(24 \text { out of } 45 \text { recruited } \\
\text { participants did not complete the study). Considering that the sample sizes are } \\
\text { small, this high attrition rate }(53.3 \%) \text { impacts on the reliability of this study's } \\
\text { results. }\end{array}$ \\
\hline Other bias & High risk & $\begin{array}{l}\text { The intervention and control groups were not comparable at baseline - the art } \\
\text { therapy group had a higher mean depression score }(P<0.01) \text { than the control } \\
\text { group. }\end{array}$ \\
\hline
\end{tabular}

Benton Fluency Task (BFT); Zarit Caregiver Burden Interview (CBI); Cornell Scale for Depression in Dementia (CSDD); Geriatric Depression Scale (GDS); Mini-Mental State Examination (MMSE); Multi Observational Scale for the Elderly (MOSES); Rivermead Behavioural Memory Test (RBMT); Tests of Everyday Attention (TEA); Wechsler Memory Scale-Revised (WMS-R)

Characteristics of excluded studies [ordered by study ID]

\begin{tabular}{|c|c|}
\hline Study & Reason for exclusion \\
\hline Camic 2014 & $\begin{array}{l}\text { Study did not compare art therapy with standard care or other psychosocial interventions for de- } \\
\text { mentia }\end{array}$ \\
\hline Fostinelli 2016 & Not a fully-published randomised controlled trial (conference poster abstract) \\
\hline Han 2010 & Intervention was not art therapy - a music therapy and activity programme was used \\
\hline Jensen 1997 & $\begin{array}{l}\text { Intervention was not restricted to art therapy - a multisensory intervention combining art, music } \\
\text { and movement was used }\end{array}$ \\
\hline Kong 2015 & Unable to confirm as a randomised controlled trial based on information available (abstract only) \\
\hline MacPherson 2009 & Intervention was not art therapy - did not involve art-making \\
\hline NHS 2012 & Intervention was not art therapy \\
\hline Odell-Miller 2006 & $\begin{array}{l}\text { Intervention was not restricted to art therapy - treatment was } 1 \text { of } 4 \text { arts therapies (music, art, dra- } \\
\text { ma, or dance-movement) }\end{array}$ \\
\hline Rusted 2002 & Not a fully-published randomised controlled trial (conference abstract) \\
\hline Rylatt 2012 & Not a randomised controlled trial \\
\hline
\end{tabular}




\begin{tabular}{ll}
\hline Study & Reason for exclusion \\
\hline Safar 2011 & Not a randomised controlled trial \\
\hline Seifert 2003 & Not a randomised controlled trial \\
\hline Stallings 2010 & Not a randomised controlled trial \\
\hline Ullan 2013 & Not a randomised controlled trial \\
\hline Walsh 2011 & Notial \\
\hline
\end{tabular}

\section{ADDITIONAL TABLES}

Table 1. TIDieR assessment

\begin{tabular}{lll}
\hline Item No & Hattori 2011 & Rusted 2006 \\
\hline Brief name & & \\
\hline 1 & $\begin{array}{l}\text { The term 'art therapy' is used to describe the in- } \\
\text { tervention. }\end{array}$ & $\begin{array}{l}\text { The term 'art therapy' is used to describe the inter- } \\
\text { vention. }\end{array}$
\end{tabular}

\section{Why}

2

More information could be provided regarding the underlying rationale for the prescriptive artmaking activities designed. Overall, the rationale, theory or goal of the elements deemed essential to the intervention could be stated more clearly.
The underlying rationale, theory or goal of the elements deemed essential to the intervention could be stated more clearly.

\section{What}

3

Materials: The materials used in delivering the intervention are clearly described.

Procedures: The intervention is reported to have "combined several techniques" which could be elaborated on. A chosen "primary task" is identified and described in further detail.
Materials: The "variety of art materials" used in delivering the intervention could be described in more detail to further define the methodology and aid replicability.

Procedures: The procedure used is reported as a "group-interactive, psychodynamic approach".

\section{Who provided}

5

Art therapy for people with dementia (Review)

Each category of intervention facilitator, their expertise and background are detailed. Their specialty backgrounds and levels of experience varied. It is unclear whether there was any specific training or a standardised protocol in terms of delivering an art therapy intervention.

\section{How}

Information is provided regarding each category of intervention facilitator and their expertise. Levels of experience are not reported. Reference is made to "induction meetings" and "handbooks" outlining the research protocol for all therapists and assistants. 


\section{Table 1. TIDieR assessment (Continued)}

Modes of delivery of the intervention and group settings (including the presence of family accompanying participants) are clearly described. There could be further reference made to expert literature or clinical experience or both to support these decisions.
Modes of delivery of the intervention and group settings are clearly described. There could be further reference to expert literature or clinical experience or both to support these decisions.

\section{Where}

The location for the intervention is described as hospital outpatient clinic setting. There could be further discussion about the choice and potential implications of this.
The location for the intervention is described as day care setting. The context of delivery of this type of intervention may be important, including environmental factors in facilitating group interaction and psychotherapy approaches. Therefore further discussion about the choice of location could be included.

\section{When and How Much}

8 The total period of time and the number, frequency and duration of intervention sessions are clearly reported. Further information regarding the underlying rationale would be valuable.
The total period of time and the number, frequency and duration of intervention sessions are clearly reported. Further information regarding the underlying rationale would be valuable.

\section{Tailoring}

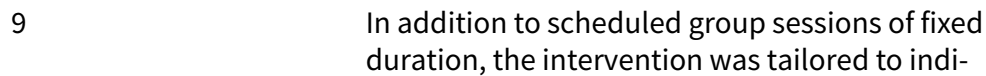
vidual ability in so much as participants were, "instructed to carry out tasks within their capability for approximately 15 minutes daily". The rationale for reported parameters as theoretically acceptable or appropriate would be valuable.

\section{Modifications}

10

\section{How well}

Some facilitators had regular supervision sessions whilst others did not. The rationale for regular supervision being offered could be more clearly stated. For example, this might be to replicate real-life practices or as a possible method of assessing, maintaining or improving fidelity.

The rationale for supervision and implications of discrepancy in attendance, could be extended beyond measuring outcomes. For example, this might include assessment of adherence and incorporating strategies to maintain or improve fidelity.

Table 2. PICO criteria - considerations for future research

\section{PICO \\ Research question components}


Table 2. PICO criteria - considerations for future research (Continued)

\section{P: Population of interest or Participants with specified type and severity of dementia problem to be addressed}

\section{I: Intervention to be consid- Art therapy as defined by the British Association of Art Therapists as a gold standard ered}

\section{C: Comparison or control in- Non-active control: no treatment, usual care, waiting list tervention \\ Active control group: attention placebo or other form of psychological intervention for dementia}

\section{0: Outcome of interest Primary outcomes}

Cognition - attention/orientation, memory, fluency, language, visuospatial perception

Affect and emotional well-being

Social functioning

Behaviour

Neuroimaging

Secondary outcomes

Adverse effects

Death

Quality of life

Economic outcomes

Carer outcomes

APPEN DICES

\section{Appendix 1. Sources searched and search strategies}

\begin{tabular}{|c|c|c|}
\hline Source & Search strategy & Hits retrieved \\
\hline \multirow{5}{*}{$\begin{array}{l}\text { 1. ALOIS (www.medi- } \\
\text { cine.ox.ac.uk/alois) } \\
\text { [Latest search } 04 \text { No- } \\
\text { vember 2016] }\end{array}$} & $\begin{array}{l}\text { art, artistic, artist, artwork, draw, drawing, paint, painting, sketch, sketching, } \\
\text { doodle }\end{array}$ & May 2014: 5 \\
\hline & & Mar 2015: 0 \\
\hline & & Jan 2016: 0 \\
\hline & & Nov 2016: 1 \\
\hline & & Oct 2017: 1 \\
\hline \multirow{3}{*}{$\begin{array}{l}\text { 2. MEDLINE In-process } \\
\text { and other non-indexed } \\
\text { citations and MEDLINE } \\
\text { 1946-present (Ovid SP) }\end{array}$} & 1. exp Dementia/ & May 2014: 25 \\
\hline & 2. Delirium/ & Mar 2015: 31 \\
\hline & 3. Wernicke Encephalopathy/ & Jan 2016: 41 \\
\hline $\begin{array}{l}\text { [Latest search } 04 \text { No- } \\
\text { vember 2016] }\end{array}$ & 4. Delirium, Dementia, Amnestic, Cognitive Disorders/ & Nov 2016: 45 \\
\hline
\end{tabular}


6. alzheimer ${ }^{\star} . \mathrm{mp}$.

7. (lewy ${ }^{\star}$ adj2 bod*).mp.

8. deliri*.mp.

9. (chronic adj2 cerebrovascular).mp.

10. ("organic brain disease" or "organic brain syndrome").mp.

11. ("normal pressure hydrocephalus" and "shunt*").mp.

12. "benign senescent forgetfulness".mp.

13. (cerebr ${ }^{\star}$ adj2 deteriorat $\left.{ }^{\star}\right) \cdot m p$.

14. (cerebral $^{\star}$ adj2 insufficient $\left.{ }^{\star}\right) \cdot m p$.

15. (pick* adj2 disease).mp

16. (creutzfeldt or jcd or cjd).mp.

17. huntington ${ }^{\star} \cdot \mathrm{mp}$

18. binswanger ${ }^{\star} \cdot \mathrm{mp}$.

19. korsako*.mp.

20. or/1-19

21. Art Therapy/

22. art.ti,ab.

23. draw.ti,ab.

24. (artist or artistic).ti,ab.

25. artwork.ti,ab.

26. (drawing* or sketching or sketches or paint ${ }^{\star}$ ).ti,ab.

27. (etch or doodle* or "still life" or tracing).ti,ab.

28. or/21-27

29. 20 and 28

30. randomized controlled trial.pt.

31. controlled clinical trial.pt.

32. random* ${ }^{\star} \cdot a b$

33. placebo.ab.

34. trial.ab.

35. groups.ab.

36. or $/ 30-35$

37. (animals not (humans and animals)).sh.

$38.36 \operatorname{not} 37$ 


\section{29 and 38}

\section{EMBASE}

1974-January 142016 (Ovid SP)

[Latest search 04 November 2016]
1. exp dementia/

May 2014: 21

2. Lewy body/

Mar 2015: 20

3. delirium/

Jan 2016: 45

4. Wernicke encephalopathy/

Nov 2016: 45

5. cognitive defect/

6. dement*.mp.

7. alzheimer ${ }^{\star} . \mathrm{mp}$.

8. (lewy* adj2 bod*).mp.

9. deliri*.mp

10. (chronic adj2 cerebrovascular).mp.

11. ("organic brain disease" or "organic brain syndrome").mp.

12. "supranuclear palsy".mp.

13. ("normal pressure hydrocephalus" and "shunt*").mp.

14. "benign senescent forgetfulness".mp.

15. $\left(\right.$ cerebr $^{\star}$ adj2 deteriorat $\left.{ }^{\star}\right) \cdot \mathrm{mp}$.

16. (cerebral $^{\star}$ adj2 insufficient $\left.{ }^{\star}\right) \cdot m p$.

17. (pick ${ }^{\star}$ adj2 disease).mp.

18. (creutzfeldt or jcd or cjd).mp.

19. huntington ${ }^{\star} \cdot \mathrm{mp}$.

20. binswanger*.mp.

21. korsako*.mp.

22. CADASIL.mp.

23. or/1-22

24. exp *art therapy/

25. art.ti,ab.

26. draw.ti,ab.

27. (artist or artistic).ti,ab.

28. artwork.ti,ab.

29. (drawing* or sketching or sketches or paint $\left.{ }^{\star}\right)$.ti,ab.

30. (etch* or doodle* or "still life" or tracing).ti,ab.

31. or $/ 24-30$

32. 23 and 31

33. randomized controlled trial/ 

34. controlled clinical trial/
35. randomly.ab.
36. placebo.ab.
37. trial.ab.
38. "single-blind*".ti,ab.
39. "double-blind"*ti,ab.
40. or/33-39
41.32 and 40

4. PSYCINFO

1806-January week 2 2016 (Ovid SP)

[Latest search 04 November 2016]

\section{1. exp Dementia/}

2. exp Delirium/

3. exp Huntingtons Disease/

4. exp Kluver Bucy Syndrome/

5. exp Wernickes Syndrome/

6. exp Cognitive Impairment/

7. dement ${ }^{\star} \cdot \mathrm{mp}$.

8. alzheimer ${ }^{\star} . \mathrm{mp}$.

9. $\left(\right.$ lewy $^{\star}$ adj2 bod $\left.^{\star}\right) \cdot \mathrm{mp}$.

10. deliri ${ }^{\star} . \mathrm{mp}$.

11. (chronic adj2 cerebrovascular).mp.

12. ("organic brain disease" or "organic brain syndrome").mp.

13. "supranuclear palsy".mp.

14. ("normal pressure hydrocephalus" and "shunt*").mp.

15. "benign senescent forgetfulness".mp.

16. (cerebr ${ }^{\star}$ adj2 deteriorat $\left.{ }^{\star}\right) \cdot \mathrm{mp}$.

17. (cerebral $^{\star}$ adj2 insufficient $\left.{ }^{\star}\right) \cdot m p$.

18. (pick* adj2 disease).mp

19. (creutzfeldt or jcd or cjd).mp.

20. huntington ${ }^{\star} . \mathrm{mp}$.

21. binswanger*.mp.

22. korsako*.mp.

23. ("parkinson^ disease dementia" or PDD or "parkinson* dementia").mp.

24. or/1-23

25. exp Art Therapy/

26. art.ti,ab.
May 2014: 4

Mar 2015: 5

Jan 2016: 5

Nov 2016: 7

Oct 2017: 8 
(Continued)

\section{7. draw.ti,ab.}

28. (artist or artistic).ti,ab.

29. artwork.ti,ab.

30. (drawing ${ }^{\star}$ or sketching or sketches or paint $\left.{ }^{\star}\right)$.ti,ab.

31. (etch* or doodle* or "still life" or tracing).ti,ab.

32. or/25-31

33. 24 and 32

34. trial.ab.

35. "single-blind*".ti,ab.

36. randomly.ab.

37. "double-blind*".ti,ab.

38. exp Clinical Trials/

39. rct.ti,ab.

40. or/34-39

41.33 and 40

42. $2014^{\star}$.up.

43. 41 and 42

5. CINAHL (EBSCOhost)

[Latest search 04 November 2016]
S1 (MH "Dementia+")

May 2014: 5

S2 (MH "Delirium") or (MH "Delirium, Dementia, Amnestic, Cognitive Disorders")

S3 (MH "Wernicke's Encephalopathy")

S4 TX dement*

S5 TX alzheimer*

S6 TX lewy* N2 bod*

S7 TX deliri ${ }^{\star}$

S8 TX chronic N2 cerebrovascular

S9 TX "organic brain disease" or "organic brain syndrome"

S10 TX "normal pressure hydrocephalus" and "shunt*"

S11 TX "benign senescent forgetfulness"

S12 TX cerebr* N2 deteriorat*

S13 TX cerebral ${ }^{\star} \mathrm{N} 2$ insufficient ${ }^{\star}$

S14 TX pick* N2 disease

S15 TX creutzfeldt or jcd or cjd

S16 TX huntington ${ }^{\star}$
Mar 2015: 0

Jan 2016: 5

Nov 2016: 10

Oct 2017: 44 
(Continued)

S17 TX binswanger*

S18 TX korsako*

$\mathrm{S} 19 \mathrm{~S} 1$ or $\mathrm{S} 2$ or $\mathrm{S} 3$ or $\mathrm{S} 4$ or $\mathrm{S} 5$ or $\mathrm{S} 6$ or $\mathrm{S} 7$ or $\mathrm{S} 8$ or $\mathrm{S} 9$ or $\mathrm{S} 10$ or $\mathrm{S} 11$ or $\mathrm{S} 12$ or $\mathrm{S} 13$ or $\mathrm{S} 14$ or $\mathrm{S} 15$ or $\mathrm{S} 16$ or $\mathrm{S} 17$ or $\mathrm{S} 18$

S20 (MH "Art Therapy")

S21 TX "art* therap*"

S22 AB art

S23 AB draw

S24 AB artist or artistic

S25 AB artwork

S26 TX drawing* OR sketching OR sketches OR paint*

S27 TX etch* OR doodle* OR "still life" OR tracing

S28 S20 OR S21 OR S22 OR S23 OR S24 OR S25 OR S26 OR S27

S29 S19 AND S28

S30 (MH "Clinical Trials")

S31 TX trial

S32 TX "single-blind*"

S33 TX "double-blind*"

S34 TX "treatment as usual"

S35 TX randomly

S36 S30 OR S31 OR S32 OR S33 OR S34 OR S35

S37 S29 AND S36

6. ISI Web of Science Core Collection [includes: Web of Science (1945-present); BIOSIS Previews (1926present); MEDLINE (1950-present); Journal Citation Reports]; BIOSIS Previews
TOPIC: (dement ${ }^{\star}$ OR alzheimer ${ }^{\star}$ OR "vascular cognitive impairment" OR "lew* bod*" OR CADASIL OR "cognit* impair*") AND TOPIC: ("art therapy" OR drawing OR sketching OR doodling OR painting OR artist OR "still life" OR "art class" OR "pen and ink") AND TOPIC: (randomly OR placebo OR groups OR trial OR RCT OR randomized OR randomised OR "double-blind" " OR "single-blind*" OR CCT OR "cross-over" OR crossover)

Search language=English
May 2014: 143

Mar 2015: 98

Jan 2016: 63

Nov 2016: 81

Oct 2017: 222

[Latest search 04 November 2016]

\section{LILACS (BIREME) "art therapy" AND dement\$}

May 2014: 0

[Latest search 04 No-

Mar 2015: 0

vember 2016]

Jan 2016: 0

Nov 2016: 0

Oct 2017: 0 
(Continued)
8. CENTRAL (via CRSO)
\#1 MESH DESCRIPTOR art therapy EXPLODE ALL TREES
May 2014: 12
[Latest search 04 No-
\#2 (artist OR artistic):TI,AB,KY
Mar 2015: 5
vember 2016]
\#3 artwork:TI,AB,KY
Jan 2016: 7
\#4 (drawing* OR sketching OR sketches OR paint $\left.{ }^{\star}\right): T I, A B, K Y$
Nov 2016: 15
\#5 (etch OR doodle* OR "still life" OR tracing):TI,AB,KY
Oct 2017: 42
\#6 \#1 OR \#2 OR \#3 OR \#4 OR \#5
\#7 dementia:MH
\#8 MESH DESCRIPTOR Dementia EXPLODE ALL TREES
\#9 dement*:TI,AB,KY
\#10 alzheimer ${ }^{\star}: T I, A B, K Y$
\#11 \#7 OR \#8 OR \#9 OR \#10
\#12 \#6 AND \#11

9. Clinicaltrials.gov

(www.clinicaltrials.gov)

[Latest search 04 November 2016] (dementia OR alzheimers OR cognition OR cognitive) AND (art OR paint OR draw OR sketch)

Filter by Interventional Studies
May 2014: 36

Mar 2015: 0

Jan 2016: 0

Nov 2016: 0

Oct 2017: 16

May 2014: 21

Mar 2015: 3

Jan 2016: 0

Nov 2016: 0

Oct 2017: 0

ISRCTN; Chinese Clini-

cal Trial Registry; Clini-

cal Trials Registry - In-

dia; Clinical Research

Information Service -

Republic of Korea; Ger-

man Clinical Trials Reg-

ister; Iranian Registry

of Clinical Trials; Japan

Primary Registries Net-

work; Pan African Clin-

ical Trial Registry; Sri

Lanka Clinical Trials

Registry; The Nether-

lands National Trial

Register]

[Latest search 04 No-

vember 2016] 
Nov 2016: 204

Oct 2017: 539

TOTAL: 1343

TOTAL after de-duplication and first-assessment by information specialists based on title and abstract

\section{CONTRIBUTIONS OF AUTHORS}

SR Deshmukh wrote the initial version of the protocol and review. JD Holmes and AG Cardno commented on the protocol and review. JD Holmes acts as the guarantor of the review.

Independently, two review authors assessed all the studies identified through the search strategy for eligibility. This was shared between the three review authors. Where necessary, we resolved any disagreement through discussion or, if required, by consulting a third party. For eligible studies, SR Deshmukh and JD Holmes independently extracted and cross-checked outcome data and assessed risks of bias for included studies using the criteria outlined in the Cochrane Handbook for Systematic Reviews of Interventions (Higgins 2011).

\section{DECLARATIONS OF INTEREST}

Sunita R Deshmukh: none known

John Holmes: none known

Alastair Cardno: none known

\section{SOURCES OF SUPPORT}

\section{Internal sources}

- No sources of support supplied

\section{External sources}

- NIHR, UK.

This review was supported by the National Institute for Health Research, via Cochrane Infrastructure funding to the Cochrane Dementia and Cognitive Improvement group. The views and opinions expressed therein are those of the authors and do not necessarily reflect those of the Systematic Reviews Programme, NIHR, NHS or the Department of Health

\section{DIFFERENCES BETWEEN PROTOCOLAND REVIEW}

The following summarised differences between protocol and review are attributed to limitations in the availability of relevant studies and data. The protocol included methods for data analysis, but it was not possible to obtain adequate data to pool in meta-analyses. We planned to separate participant data into prespecified subgroups listed in the protocol, in order to check for important comparisons between them, but insufficient data are currently available for this.

The following summarised differences between protocol and review are attributed to changes made to the review to incorporate new formats and requirements for Cochrane Reviews. We used the GRADE approach in interpreting findings (Langendam 2013; Schünemann 2013) and created a 'Summary of findings' table. 
INDEX TERMS

\section{Medical Subject Headings (MeSH)}

Art Therapy [ ${ }^{\star}$ methods]; Dementia [ ${ }^{\star}$ therapy]; Randomized Controlled Trials as Topic; Recreation Therapy

\section{MeSH check words}

Humans 